\title{
Author Correction: Explainable machine learning models of major crop traits from satellite-monitored continent-wide field trial data
}

\author{
Saul Justin Newman iD and Robert T. Furbank iD
}

Correction to: Nature Plants https://doi.org/10.1038/s41477-021-01001-0, published online 4 October 2021.

The Grains Research and Development Corporation have made us aware of concerns they have about possible data probity issues pertaining to reuse of the web accessed data of their field trials we utilized to ground truth and build the models. As such, we amend the Data availability section to address these concerns as follows.

\section{Data availability}

All data are available from the Supplementary Information, the linked database descriptor publication ${ }^{5}$ uploaded to Scientific Data and the figshare ${ }^{7}$ repository, after screening under our own extensive imputations and quality controls and freely available for research or non-commercial purposes under a CC-BY-NC 3.0 license. The dataset which forms the basis (in whole or part) for this paper is based predominantly on data sourced from the Grains Research \& Development Corporation (GRDC) and GRDC's extensive investment in the collection, development and presentation of that dataset is acknowledged. GRDC did not authorise the reproduction, publication or communication of the dataset. The dataset has not been subject to GRDC's quality control processes, and does not include updates and corrections that have been made to the dataset and as such may be unreliable. Results of research based on the dataset should not be relied on for any purpose. Any person wishing to conduct research using National Variety Trials (NVT) data must approach GRDC directly with a research proposal, noting that terms and conditions may apply. Alternately, the extensively quality-controlled and imputed Figshare repository remains freely accessible for research under a creative commons license ${ }^{7}$.

The changes have been made to the html and PDF versions of the article.

Published online: 11 January 2022

https://doi.org/10.1038/s41477-022-01096-Z

(C) The Author(s), under exclusive licence to Springer Nature Limited 2022 